\title{
Postresponse exposure to warning signal in avoidance extinction*
}

\author{
DENNIS J. DELPRATO \\ Eastern Michigan University, Ypsilanti, Michigan 48197
}

\begin{abstract}
Delayed termination of the warning signal following extinction responses has been shown to facilitate extinction of discriminative avoidance. In order to determine the relative roles in extinction of delayed termination per se and postresponse exposure to the warning signal, which is necessarily confounded with delayed termination treatment, seven groups of rats were first trained on a one-way avoidance task in which a buzzer served as part of the warning-signal complex. Then, on nonshock extinction trials, the buzzer (a) terminated immediately with a response and was not reinstated in the postresponse interval, (b) terminated immediately with a response and was reinstated for a 5- or 10 -sec period 5 or 15 sec following the response, or (c) terminated 5 or $10 \mathrm{sec}$ following a response (delayed termination). Results indicated that exposure to the buzzer at postresponse intervals greater than 5 sec following responses was critically involved in reducing resistance to extinction. This finding supports a conditioned relief interpretation of the delayed warning signal termination effect and is consistent with the effect of response prevention techniques on extinction.
\end{abstract}

Katzev has systematically investigated a method of carrying out extinction of discriminative avoidance that involves delayed termination of the warning signal following extinction responses. Previous work has shown that the rate of extinction of two-way (Katzev, 1967) and one-way (Delprato, 1969) avoidance is positively related to the duration of the delay in warning signal termination. Since the technique of delayed warning signal offset necessarily involves exposing $S$ to the warning signal in the postresponse interval, it is possible that such exposure, rather than delayed termination per se, is the primary empirical variable underlying the facilitation of extinction associated with this procedure. The centrality of exposure in extinction has been convincingly demonstrated by several experiments in which exposure to a conditioned aversive stimulus in the absence of primary aversive stimulation has been an efficacious technique for the elimination of fear and avoidance behavior (e.g., Baum, 1970; Delprato \& Jackson, in press).

The main purpose of the present experiment was to determine the relative roles of postresponse exposure to the warning signal and delayed termination per se in the delayed warning signal termination effect. This was accomplished by comparing extinction performance of groups experiencing delayed signal termination with that of groups experiencing immediate response-contingent signal termination plus reinstatement of the signal, with postresponse exposure duration of the signal equivalent in the two conditions. If delayed signal termination facilitates extinction, faster extinction should occur in delayed termination groups than in immediate termination plus reinstatement conditions. On the other

*Supported by a National Science Foundation institutiona grant to Eastern Michigan University. The author thanks John Bonifas for his assistance in collection of the data. Requests for reprints should be sent to Dennis J. Delprato, Department of Psychology, Eastern Michigan University, Ypsilanti. Michigan 48197 . hand, to the extent that exposure to the signal postresponse can account for the delayed termination effect, delayed termination should be no more effective than immediate termination plus reinstatement when postresponse exposure duration of the signal is held constant.

A secondary purpose of the study was to evaluate an interpretation of the delayed warning signal termination effect that emphasizes exposure to the warning signal in the postresponse interval. The conditioned relief analysis of the effect is based on Denny's relaxation theory (Denny, 1971; Denny \& Ratner, 1970). Denny has identified two independent events associated with the nonshock period in escape and avoidance: relaxation and relief. Relaxation response is a relatively long-latency event that occurs following termination of primary and conditioned aversive stimuli. Relaxation is critical in mediating the approach component of avoidance acquisition and in avoidance extinction. Relief also occurs following termination of primary and conditioned aversive stimuli. Relief, like relaxation, seems critical in acquisition and extinction of avoidance, but it is a much shorter latency response.

As already indicated, the conditioned relief analysis of the delayed warning signal effect suggests that the mere presence of the warning signal postresponse in extinction is critical. According to this view, delayed signal termination and immediate termination followed by reinstatement of the signal will facilitate avoidance extinction to the extent that exposure to the signal is paired with relief postresponse. There is nothing unique about delayed termination per se; if the signal is present at such time postresponse that it occurs in close contiguity with relief, then relief should be conditioned to the signal. Conditioned relief then competes with fear and avoidance to facilitate extinction of the avoidance response. Since onset of relief is presumably not simultaneous with performance of the avoidance 


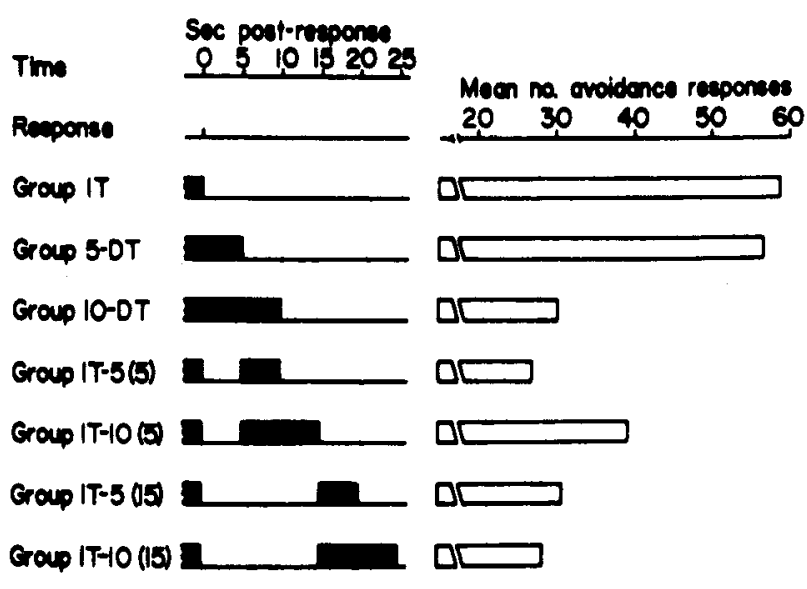

Fig. 1. The relationship between an extinction response and buzzer exposure on each trial in the various groups is depicted in the left half. For example. in Group IT (immediate termination) the buzzer was on prior to the response (as it was in all conditions), it terminated with the response, and it remained off throughout the postresponse interval. The right half shows the mean number of responses with latencies less than the avoidance interval of $5 \mathrm{sec}$ (avoidance responses) of each group during the 75 nonshock extinction trials.

response during extinction, short delays in signal termination which expose $S$ to the signal postresponse prior to the onset of relief ( $5 \mathrm{sec}$ in the present study) should not be as effective as immediate signal termination with reinstatement of the signal 5 or $15 \mathrm{sec}$ later for the same 5-sec duration if reinstatement results in signal exposure occurring in closer relationship with relief. However, on the basis of evidence that indicates relief may have a $2-5-\mathrm{sec}$ latency postresponse (Delprato \& Denny, 1968), longer signal durations (e.g., $10 \mathrm{sec}$ ) following the extinction response should permit conditioning to the signal with both delayed termination and immediate termination with reinstatement 5 or $15 \mathrm{sec}$ later, since the longer signal duration would be more likely to overlap relief when the signal is delayed in offset and presented in the first $10 \mathrm{sec}$ of the postresponse interval.

\section{METHOD}

\section{Subjects}

The Ss, 70 male Sprague-Dawley rats, were obtained from Spartan Research Animals, Haslett, Michigan. They weighed 316-505 g and were experimentally naive.

\footnotetext{
Apparatus

The apparatus had one white and one black $45.7 \times 11.4 \mathrm{x}$ $34.3 \mathrm{~cm}$ chamber, and a manually operated opaque guillotine door separated the two chambers. The entire floor was constructed of $.32-\mathrm{cm}$-diam stainless steel rods, $1.59 \mathrm{~cm}$ apart, center to center. A $1.0-\mathrm{mA}$ scrambled shock from a Grason-Stadler E1064GS shock generator was delivered to the black (shock) side. An insulated door buzzer that raised the sound level in each compartment from 45 to $68 \mathrm{~dB}$ (Bruel and Kjaer sound-level meter, A-scale) was attached to the center of the rear of the apparatus.
}

\section{Procedure}

Following $4 \mathrm{~m}$ in of acclimation to the apparatus, each $\mathrm{S}$ was trained in one-way avoidance to an acquisition criterion of three consecutive avoidance responses. On each trial, $\mathrm{S}$ was placed in the center of the shock compartment, facing the door, and $2 \mathrm{sec}$ later buzzer onset and opening of the guillotine door signaled that shock onset was to occur in $5 \mathrm{sec}$ (avoidance interval). On escape trials, both buzzer and shock were response terminated when the full body of $S$ crossed the midline of the apparatus: the same response immediately terminated the buzzer on avoidance trials. The door was closed immediately following a response on all trials. The $S$ spent a total of $40 \mathrm{sec}$ in the safe compartment, after which it was placed back into the shock side for the next trial.

After $\mathrm{S}$ met acquisition criterion, it was placed back into the shock side as usual and nonshock extinction began (all Ss). Seven groups of $10 \mathrm{Ss}$ were formed in an unbiased manner. The procedure in extinction was the same as acquisition except as indicated below. The left half of Fig. 1 depicts the relationship between an extinction response and buzzer exposure on each trial in the seven independent groups. Group IT (immediate termination) continued to experience immediate response-contingent buzzer termination; this group never received the buzzer in the postresponse interval. Groups 5-DT ( $5 \mathrm{sec}$ delayed buzzer termination) and 10-DT (10 sec delayed buzzer termination) experienced a 5 - and 10-sec delay in buzzer termination, respectively, following each extinction response. The remaining four groups experienced immediate response-contingent buzzer termination (IT) plus reinstatement of the buzzer on each trial. In Group IT-5 (5), the buzzer was reinstated for a duration of $5 \mathrm{sec}$, beginning $5 \mathrm{sec}$ after it had terminated. Group IT-10 (5) had the buzzer reinstated for a duration of $10 \mathrm{sec}$, onset occurring $5 \mathrm{sec}$ after it had terminated. Groups IT-5 (15) and IT-10 (15) experienced buzzer durations of 5 and $10 \mathrm{sec}$, respectively, with onset $15 \mathrm{sec}$ after response-contingent termination. The $\mathrm{Ss}$ were run for a total of 75 extinction trials or until failure to respond within $30 \mathrm{sec}$ after trial onset for 10 consecutive trials. When a failure to respond occurred, the guillotine door was lowered and the buzzer terminated; $S$ then spent the 40 -sec intertrial interval in the shock compartment prior to onset of the next trial. The buzzer was not presented during the intertrial interval following no response trials.

\section{RESULTS AND DISCUSSION}

The mean number of shock escape trials in acquisition was 3.7 (the groups means ranged from 3.0-4.9). An analysis of variance showed that the between-group variability was negligible $(\mathrm{F}=1.01)$, indicating that the groups were comparable in avoidance performance prior to differential treatment.

The extinction data are presented in the right half of Fig. 1. The dependent variable is the mean number of responses with latencies less than $5.0 \mathrm{sec}$ (avoidance responses). Comparison of Groups IT, 5-DT, and 10-DT should show the delayed termination effect. The effect was obtained in that resistance to extinction significantly varied among these groups $(\mathrm{F}=3.67 \mathrm{df}=$ $2 / 27, p<.05$ ). It is important to note, however, that facilitation of extinction was obtained with only the $10-\mathrm{sec}$ delay in buzzer termination (Group IT vs Group 10-DT, $p<.05$ ). The group with 5 -sec delay in buzzer termination was very similar to the immediate termination condition. 
An analysis of variance over all seven groups indicated significant between-group variability $(\mathrm{F}=2.42$, df $=$ $6 / 63, p<.05$ ). Individual comparisons between pairs of means followed Lindquist (1953, p. 93).

The present results strongly suggest that delayed buzzer termination per se is of much less importance than buzzer exposure postresponse that necessarily results with delayed termination. This conclusion is especially supported when extinction performance of Groups IT-5 (5) and IT.5 (15) is compared with that of Group 5-DT. These three groups all had 5-sec postresponse exposures to the buzzer, and the former two groups experienced immediate buzzer termination. In contrast to the hypothesis that delayed termination, apart from postresponse buzzer exposure, facilitates extinction, Group 5-DT was not less resistant to extinction than the groups with immediate termination plus reinstatement [Groups IT-5 (5) and IT-5 (15)]. Indeed, these two groups, administered immediate termination with a 5 -sec period of buzzer reinstatement 5 or $15 \mathrm{sec}$ later, were significantly less resistant to extinction than Group 5-DT $(p<.05)$.

This latter finding, coupled with the fact that Groups IT-5 (5) and IT-5 (15), but not Group 5-DT, were significantly less resistant to extinction than Group IT $(\mathrm{p}<.05)$, is consistent with the conditioned relief interpretation outlined above. According to this interpretation, exposure to the buzzer in the first $5 \mathrm{-sec}$ interval postresponse (Group 5-DT) was not more effective than immediate termination without reinstatement in eliminating avoidance, because relief was not of sufficient magnitude soon after a response. Rather, relief is a postresponse phenomenon that is not simultaneous with response. Therefore, 5-sec buzzer exposures only led to the conditioning of relief to the buzzer when it was presented sometime beyond $5 \mathrm{sec}$ postresponse.

The performance of the three groups with 10 -sec buzzer exposures postresponse also points to the importance of exposure rather than delayed buzzer termination as such in extinction behavior. Group 10-DT showed the delayed termination effect when compared with Groups IT and 5-DT; however, Groups IT-10(5) and IT-10 (15), though experiencing immediate buzzer termination, were not more resistant to extinction than Group 10-DT ( $>$.05). Since immediate buzzer termination followed by a $10-\mathrm{sec}$ period of reinstatement was sufficient to produce extinction behavior comparable to $10-\mathrm{sec}$ delayed termination treatment, it seems appropriate to attribute the extinction performance of Group 10-DT relative to Groups IT and 5-IT more to the $10-\mathrm{sec}$ postresponse buzzer exposures than to delayed termination per se.

Furthermore, the conditioned relief interpretation of the mechanism by which postresponse buzzer exposure operates to affect extinction is sufficient to account for the equivalent performance of Groups 10.DT. IT-10 (5). and IT-10 (15). as well as the significantly accelerated extinction of Groups 10-DT and IT-10 (15) relative to Group 5-DT $(p<.05)$. As predicted, the data of the three groups with 5-sec buzzer exposures postresponse suggest that buildup of relief was such that the buzzer had to be presented after the fifth second postresponse for relief to be conditioned to the buzzer. The three groups with 10-sec postresponse buzzer exposure experienced the buzzer after the fifth second postresponse, but Group 5-DT did not. The equivalent extinction behavior of all three groups with $10-\mathrm{sec}$ buzzer exposures postresponse and the reduced resistance to extinction of two of these groups relative to Group 5-DT indicate that once relief built up after $5 \mathrm{sec}$ postresponse, it remained optimal. It should be noted that while Group IT-10(5) responded considerably less than Group 5-DT, as the conditioned relief analysis requires, the difference between these two groups failed to reach significance at the .05 level.

The main finding of the present study is that the delayed termination effect (Katzev, 1967: Delprato. 1969 ) is primarily the result of postresponse exposure to the warning signal rather than delayed termination per se. The effect of delayed warning signal termination on avoidance extinction can now be viewed as operating in much the same way as that of the method of response prevention in which the $S$ if forced to remain in the presence of the conditioned aversive stimulus (Baum, 1970). At an operational level, both delayed signal termination and response prevention facilitate extinction by exposing the $S$ to the conditioned aversive stimulus in the absence of primary aversive stimulation. There is no one completely acceptable theoretical interpretation of the mechanism by which exposure operates to effect extinction of avoidance in these two methods. The results and conditioned relief interpretation of the present experiment, however, are in agreement with Baum's (1972) finding that response prevention was more effective when rats were given a delay or cooling-off period following acquisition and prior to treatment than when response prevention immediately followed acquisition. Baum's interpretation of these results made use of Denny's (1971) postulated long-latency version of relief, i.e., relaxation, because of the relatively long delay interval used; otherwise, Baum's analysis was virtually identical to that proposed for the present data.

We should note that the present results are not necessarily as clear-cut in demanding a conditioned relief, as opposed to conditioned relaxation, interpretation as one might like. Recent data of Tortora and Denny (1973) indicate that relief occurs only after the removal of a conditioned aversive stimulus when the shock level during fear conditioning is very high $(1.8 \mathrm{~mA})$ or when a large number of fear conditioning trials is given with a weaker shock. Since we used a shock level considerably lower than $1.8 \mathrm{~mA}(1.0 \mathrm{~mA})$ and a mean of only 3.7 escape (fear conditioning) trials was needed in acquisition. it may be that conditioned 
relaxation is the appropriate explanatory concept here. This degree of inconclusiveness regarding relief and relaxation is difficult to resolve since they are assumed to have similar behavioral effects.

One aspect of our methodology that could be interpreted as detracting from the above conclusions deserves comment. Specifically. one might argue that the buzzer was very redundant and acquired only minimal control over avoidance in the one-way task. Handling, apparatus cues, and raising of the guillotine door might have been the primary avoidance cues. This argument loses force in light of Thompson and Hjelle's (1965) results, which indicate a considerable controlling effect of a buzzer on a one-way task almost identical to the one used here.

\section{REFERENCES}

Baum, M. Extinction of avoidance responding through response prevention (flooding). Psychological Bulletin, 1970, 74, 276-284.

Baum, M. Flooding (response prevention) in rats: The effects of immediate vs delayed flooding and of changed illumination conditions during flooding. Canadian Journal of Psychology, $1972,26,190-200$.
Delprato, D. J. Extinction of one-way avoidance and delayed warning-signal termination. Journal of Experimental Psychology, 1969, 80, 192-193.

Delprato, D. J., \& Denny, M. R. Punishment and the length of non-shock confinement during the extinction of avoidance. Canadian Journal of Psychology, 1968, 22, 456-464.

Delprato, D. J., \& Jackson, D. E. Counterconditioning and exposure-only in the treatment of specific (conditioned suppression) and generalized fear in rats. Behaviour Research \& Therapy, in press.

Denny, M. R. Relaxation theory and experiments. In F. R. Brush (Ed.), Aversive conditioning and learning. New York: Acad emic Press, 1971.

Denny, M. R., \& Ratner, S. C. Comparative psychology. (Rev. ed.) Homewood, Ill: Dorsey, 1970.

Katzev, R. Extinguishing avoidance responses as a function of delayed warning signal termination. Journal of Experimental Psychology, 1967, 75, 339-344.

Lindquist. E. F. Design and analysis of experiments in psychology and education. Boston, Mass: Houghton Mifflin, 1953.

Thompson, R. W., \& Hjelle, L. A. Effects of stimulus and response complexity on learning under bilateral spreading depression. Journal of Comparative \& Physiological Psychology, 1965, 59, $122-124$.

Tortora, D. F.. \& Denny, M. R. Flooding as a function of shock level and length of confinement. Learning \& Motivation, $1973,4,276-283$.

(Received for publication August 28, 1973; revision accepted October 11,1973 .) 